\title{
Investigation of the potential impact of storage place on tissue free water tritium and organically bound tritium activity determination
}

\author{
N. Baglan ${ }^{1, *}$, E. Ansoborlo ${ }^{2}$, C. Cossonnet ${ }^{3}$, Y. Losset ${ }^{4}$ and M. Crozet ${ }^{2}$ \\ ${ }^{1}$ CEA/DAM/DIF, 91297 Arpajon, France. \\ 2 CEA/DEN/DRCP/CETAMA, BP17171, 30207 Bagnols-sur-Cèze, France. \\ 3 IRSN/PRP-ENV/STEME/LMRE, bât 501, Bois des Rames, 91400 Orsay, France. \\ ${ }^{4}$ CEA/DAM/CVA, 21120 Is-sur-Tille, France.
}

Received: 17 March 2017 / Accepted: 26 July 2017

\begin{abstract}
When focusing on environmental tritium measurement, water is the most common matrix, and monitoring is mainly concerned by tissue free water tritium (TFWT) and organically bound tritium (OBT) analysis. Moreover, in order to improve knowledge on tritium behaviour and migration, TFWT and OBT determinations are performed at low activity level. TFWT and OBT determinations could also be performed after incidental releases to determine potential impact to the environment and to man. As hydrogen isotopes are prone to isotopic exchange with atmospheric moisture, the key point when analysing TFWT and OBT is to ensure appropriate preservation of the sample from its sampling to its analysis.

Aiming to answer to the following question: does the storage condition could change activity level within the sample? An interlaboratory exercise was organized within the framework of a French tritium working group to follow both TFWT and OBT activity scrutinizing for any variation on a one-year period. For this study about $50 \mathrm{~kg}$ of grass were sampled, homogenized, packaged and frozen prior dispatching to the participants. Six aliquots were sent to each participating lab to determine both TFWT and OBT activity concentrations, starting when all the labs received the sample, continuing two weeks, one month, three months, six months and one year after to check for any variation in the measured activity concentration. The results were treated for each analysis date, for each participating lab and considering the overall population by calculating the mean and associated standard deviation. As all values are consistent, the main conclusion of this work is that TFWT and OBT activity concentrations remain constant when appropriate storage conditions are used. This point is of importance as sample conservation is always a critical parameter for tritium determination in environmental samples and also when considering the fabrication of certified reference materials.
\end{abstract}

Keywords: tritium fractions / analysis / storage / inter-laboratory exercise

\section{Introduction}

Tritium has a number of anthropogenic sources which account for the dominant proportion of the global tritium inventory. These tritium sources include fallout from previous nuclear weapons testing, nuclear reactors, future fusion reactors, fuel reprocessing plants, heavy water production facilities and commercial production for medical diagnostics, radiopharmaceuticals, luminous paints, sign illumination, self-luminous aircraft, airport runway lights, luminous dials, gauges, wrist watches, and others (ASN, 2010). All these licensed nuclear facilities are releasing tritium in the environment where it can

*Corresponding author: nicolas.baglan@cea.fr exist in several forms, such as gaseous (HT, $\left.\mathrm{HTO}, \mathrm{CH}_{3} \mathrm{~T}\right)$, liquid (HTO or organic molecules in solutions) or organically bound tritium (OBT) which can become incorporated into living organisms (vegetables, animals, humans) (Kim et al., 2013). Given these complexities and the probability of increasing concentrations, it is necessary to be able to accurately determine the various species of tritium in the environment for public and regulatory insurance. OBT has become of increased interest within the last decade, with a specific focus on its behaviour and also its analysis, which are important to assess tritium distribution and fate in the environment (AGIR, 2007; Baglan et al., 2013).

One important tritium property is its capacity to exchange with all other hydrogen isotopes in all living organisms with a large predominance of short kinetics (König, 1990; 
Belot et al., 1996; Brudenell et al., 1997; Keum et al., 2006). This point, which plays a major role to explain tritium fate in the environment, is also an analytical concern. Indeed, the analytical procedure used to determine TFWT and OBT activity concentrations consists in numerous stages where contaminations could occur (Pointurier et al., 2004; Baglan et al., 2010; CETAMA 2013). Therefore, analytical procedure should be robust enough to avoid any modification or external contamination of the sample during its residence time in the lab. Indeed, sample can be stored one month or more to analyse the activity concentration determination, delay which is long enough regarding to tritium exchange kinetics. Moreover, for expertise purposes several labs with different tritium atmosphere depending on the closeness of any discharging facility, the distance from the sea could be mandated to realize the same analysis.

For all these reasons, it was decided to organize an intercomparison exercise where TFWT and OBT activity concentrations determination should be performed on a regular basis on a one-year period. As such, an exercise required to send fresh sample to participating labs registration was limited to French labs to avoid any trouble in sending natural sample. The chosen matrix was grass due to the relative easiness of sampling at a $50 \mathrm{~kg}$ scale.

Once in the lab, the samples were stored according to each lab storage procedure before treatment. The treatment where defined at the beginning of the exercise to allow each determination to be consistent and therefore compared. All the results obtained along this exercise were gathered and a statistical treatment was performed. To get preliminary information, mean per lab, per day of analysis and overall mean with their associated standard deviation were compared. Afterwards, an analysis of variance was performed to look for minor effects not revealed otherwise, these effects being evaluated regarding technical information available from the participants.

The results obtained are satisfactory as on a one-year period, no significant difference is observed either for TFWT or OBT determination. Moreover, trends, which could be hypothesised from the statistical treatment, originate from experimental and/or technical sources.

\section{Experimental}

\subsection{Sample preparation, delivery and storage}

In June 2013, about $50 \mathrm{~kg}$ of grass were sampled and homogenized. Sealed aliquots of about $300 \mathrm{~g}$, which should be sufficient to each participating lab for both TFWT and OBT determination, were prepared before freezing. Once frozen $\left(T \approx-18^{\circ} \mathrm{C}\right)$ six aliquots were sent to each participating lab through refrigerated truck.

TFWT and OBT were analysed six times over a one-year period according to the following schedule, two days after sample delivery $\left(t_{1}=t_{0}+2 \mathrm{~d}\right)$, two weeks after $\left(t_{2}=t_{0}+15 \mathrm{~d}\right)$, one month after $\left(t_{3}=t_{0}+1 \mathrm{~m}\right)$, three months after $\left(t_{4}=t_{0}+3 \mathrm{~m}\right)$, six months after $\left(t_{5}=t_{0}+6 \mathrm{~m}\right)$ and one year after delivery $\left(t_{6}=t_{0}+1 \mathrm{y}\right)$. Storage conditions were not fixed for this exercise to allow observing for potential bias.

Anyway, during this one-year period, all the labs have stored the sealed samples in freezer without any modification.

\subsection{Participants}

Nine labs, numbered from 1 to 9 , were registered for this exercise aiming to investigate the influence of storage on TFWT or OBT activity concentration determination in environmental samples. Eight labs have sent their results before the deadline, only lab 9 did not. Therefore, statistical treatment was only performed for the eight labs replying before the deadline.

Participating labs belong to different organism, AREVA (1), CEA (4), EDF (1), IRSN (1), Marine Nationale (1) and University (1) with a common interest on environmental monitoring.

\subsection{Sample analysis}

The general scheme to extract TFWT or OBT from an environmental matrix is summarized on Figure 1.

As far as TFWT analysis is concerned, dehydration was performed through freeze-drying for six labs out of eight. The two others were using derived methods with temperature of the cold (about $5^{\circ} \mathrm{C}$ ) and hot points (between 30 and $40^{\circ} \mathrm{C}$ ) which could differ. Dehydration water was recovered and mixed with UltimaGOL LLT ${ }^{*}$ before measurement by liquid scintillation counting (LSC) for seven labs while the last one which is using mass spectrometry stored the dehydration water under vacuum after outgassing residual air (helium).

For OBT analysis, the dehydrated samples are sealed under vacuum before measurement for the lab using mass spectrometry and combusted in a tubular oven (5 labs) or using an Oxydiser (2 labs). Therefore, depending on the combustion apparatus UltimaGOLD LLT ${ }^{\circledR}$ or Monophase ${ }^{\circledR} \mathrm{S}$ are used for LSC counting.

To allow a complete overview of the procedure in each lab, several technical indicators such as water content, fresh to dry mass ratio and hydrogen percentage were also discussed.

\subsection{Statistical processing method}

The statistical tests classically used to interpret the results of a round-robin refer to a population meeting the criteria of a normal distribution defined by the mean and the variance. To check this specific point, the Shapiro-Wilk test was used.

For this exercise aiming to investigate the potential influence of storage conditions on TFWT and OBT determination on environmental samples, a simplified approach was first chosen. After checking the results obtained per aliquot or per lab for outliers (Grubbs, Dixon tests) and homoscedasticity (Bartlett test), only descriptive statistic (location and dispersion) was used. The criteria chosen to guarantee consistency were the overlapping of the interval where $95 \%$ of the results are found for each set of data (lab, aliquots, overall). Therefore, the mean per lab and per aliquot plus and minus twice their standard deviation were compared to the overall mean plus and minus twice its associated standard deviation.

To strengthen the conclusions issued from this simple comparison, a statistical analysis was performed. For that, an ANAlysis Of VAriance (ANOVA) was done using an F-test to check the null hypothesis (distribution statistically equivalent with or without the impact of the studied factor, the studied 


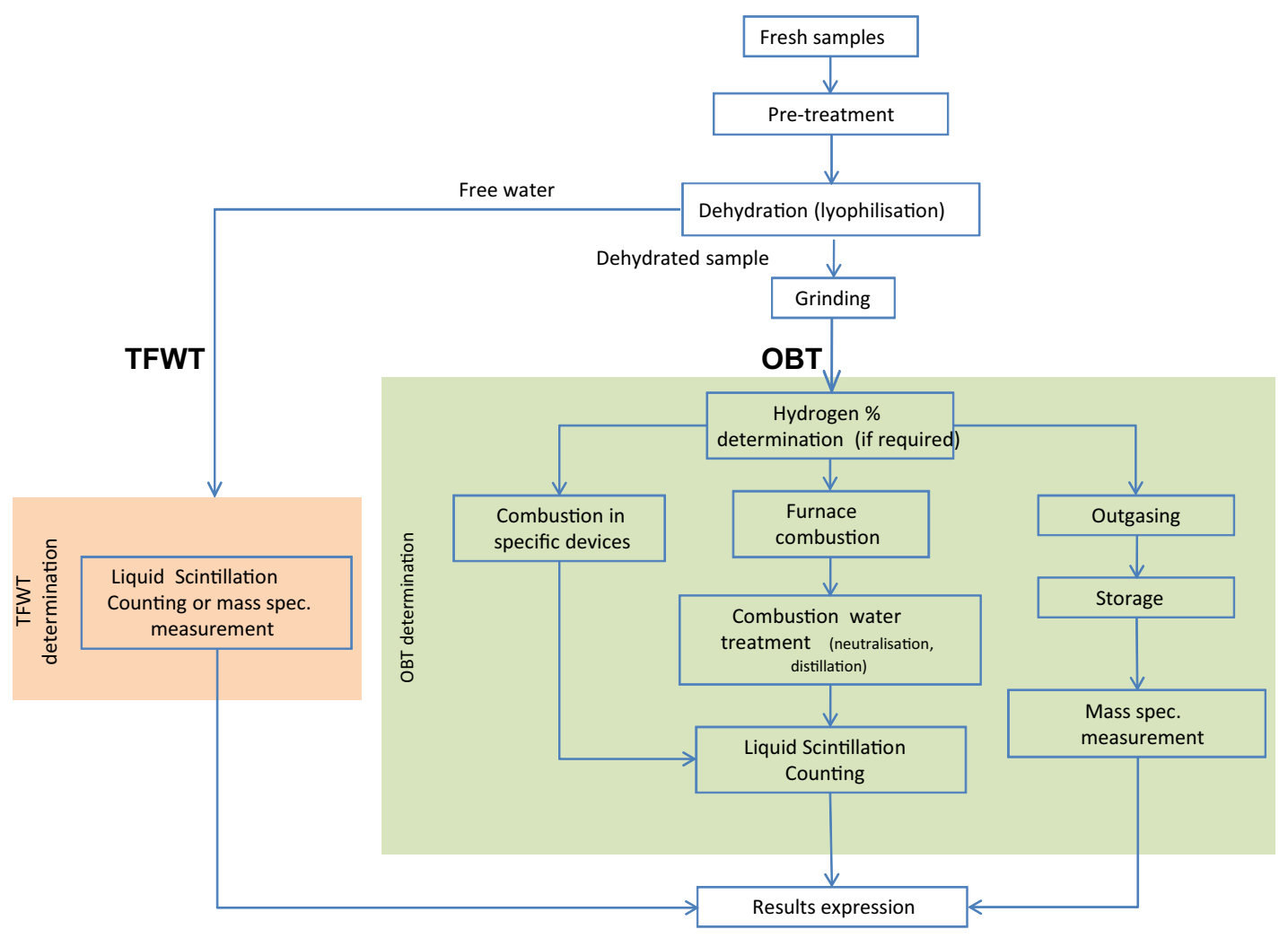

Fig. 1. TFWT and OBT analytical scheme for environmental matrixes.

factor can be the lab or the aliquot). In addition, a tendency analysis was done to check for any variation within a lab along time using (time is then the studied factor in ANOVA). The calculations for ANOVA analysis were performed by using the JMP_ 12.0.0 (Statistical Discovery) from SAS Institute Inc (http://www.jmp.com/software/).

\section{Results and discussion}

Aiming to provide the most comprehensive overview of the results issuing for this interlaboratory exercise, the consistency of the technical indicators will be discussed, the results of preliminary testing presented before to come to TFWT and OBT results. In this part, all the uncertainties are given for a confidence level of about $95 \%(k=2)$. When only statistical variations are considered to evaluate results consistency, the means plus or minus twice the standard deviation are compared.

\subsection{Technical indicators}

The values determined for both water content $(67.4 \pm 2.4) \%$ and fresh to dry mass ratio $(3.10 \pm 0.16)$ are consistent with environmental values. Moreover, they are also consistent within the labs for all the aliquots and also between labs with relative associated uncertainty of $3.5 \%$ and $5.2 \%$ respectively, demonstrating the ability of the labs involved in this exercise to realize the dehydration stage.

On the same way, the mean hydrogen percentage $(6.06 \pm 0.14) \%$ is consistent between labs and with the cellulose $\left[\left(\mathrm{C}_{6} \mathrm{H}_{10} \mathrm{O}_{5}\right)_{n}\right]$, one of the main components of grass samples, calculated value of $6.2 \%$.

\subsection{Preliminary testing}

Some important points were checked before the treatment: (i) the presence of outliers was investigated for TFWT and OBT activity concentrations considering both lab's individual values and the results obtained for each date of analysis; (ii) considering the results provided by the labs together, the normality of the distribution was checked using the Wilcoxon test; (iii) the homogeneity of the variance was checked using the Cochran test for all labs;

(i) Outliers were only detected for OBT determination for both combustion water and dry grass results, the values determined in this lab are discarded from the statistical evaluation.

(ii) The normality of the distribution was assessed for all sets of data (Bq. $\mathrm{L}^{-1}$ of dehydration water, Bq. $\mathrm{L}^{-1}$ combustion water and Bq. $\mathrm{kg}^{-1}$ of dry grass).

(iii) For each set of data the variances are homogeneous.

Therefore, both mean comparison and analysis of variance (ANOVA) could be performed.

For both tritium fractions (TFWT, OBT) the data were exploited identically, the mean results obtained by each lab for the different analysis day and at each analysis day date for all labs were first compared to the overall mean to determine the main trends.

In addition to that, a statistical evaluation was done through an ANOVA analysis to check for any factor for which the impact couldn't be observed by simply performing a raw mean comparison. 
Table 1. TFWT activity concentrations (Bq. $\mathrm{L}^{-1}$ ) determined in each lab for each analysis time plus associated mean ( $\mathrm{m}$ ) and two times the standard deviation (2s) per lab and per day.

\begin{tabular}{|c|c|c|c|c|c|c|c|c|c|}
\hline & $t_{0}+2 \mathrm{~d}$ & $t_{0}+15 \mathrm{~d}$ & $t_{0}+30 \mathrm{~d}$ & $t_{0}+90 \mathrm{~d}$ & $t_{0}+180 \mathrm{~d}$ & $t_{0}+360 \mathrm{~d}$ & m (lab) & $2 \mathrm{~s}$ & $2 \mathrm{~s} / \mathrm{m}(\%)$ \\
\hline Lab 1 & 29.6 & 29.7 & 29.6 & 29.0 & 29.5 & 28.5 & 29.3 & 0.9 & 3.2 \\
\hline Lab 2 & & & & 33.9 & 30.6 & 31.6 & 32.0 & 3.4 & 10.6 \\
\hline Lab 4 & 30.4 & 29.0 & 35.5 & 26.7 & 26.9 & 31.0 & 29.9 & 6.5 & 21.7 \\
\hline Lab 5 & 24.5 & 25.7 & 26.2 & 25.3 & 25.6 & 26.0 & 25.6 & 1.2 & 4.7 \\
\hline Lab 8 & 28.9 & 29.2 & 28.6 & 28.6 & 30.9 & 29.7 & 29.3 & 1.8 & 6.0 \\
\hline m (day) & 29.3 & 28.3 & 29.6 & 28.9 & 29.1 & 29.5 & & & \\
\hline $2 \mathrm{~s}$ & 6.1 & 3.0 & 6.1 & 5.0 & 4.0 & 7.8 & & & \\
\hline $2 \mathrm{~s} / \mathrm{m}(\%)$ & 21 & 11 & 21 & 17 & 14 & 27 & & & \\
\hline
\end{tabular}

\subsection{Tissue free water tritium (TFWT)}

Considering TFWT, activity concentration values could be expressed both in Bq. $\mathrm{L}^{-1}$ (or Bq. $\mathrm{kg}^{-1}$ ) of dehydration water or Bq. $\mathrm{kg}^{-1}$ of fresh sample, assuming that the fresh sample to dehydration water mass ratio is constant. Therefore, the observations made for TFWT activity concentration on dehydration water are also valid for the fresh material.

Uncertainties obtained by labs for each individual measurement are not quoted in Table 1 where only individual values, mean and standard deviation are presented. In this exercise, for a confidence level of about $95 \%(k=2)$, the relative uncertainties provided for each individual value by participating labs vary from 4 to $18 \%$.

All the data obtained during this one year exercise are summarized in Table 1 for each lab and each analysis day.

Using all the data summarized in Table 1 allows determining the overall mean activity concentration value plus or minus twice its standard deviation: $\overline{\overline{\mathrm{C}_{\mathrm{A}}}}=(29.1 \pm 5.3) \mathrm{Bq} \cdot \mathrm{L}^{-1}$.

For all the labs the mean TFWT activity concentration calculated from the results obtained at each measurement time are consistent with the overall one. Therefore, storage conditions in all participating lab seem to be appropriate for sample conservation as far as TFWT analysis is concerned. Moreover, for the two labs which are not using freeze-drying but a derived method (lab 3 and 4 ) the associate dispersion is higher confirming the efficiency and suitability of freezedrying for environmental sample dehydration.

Whatever the time after sampling is, the mean TFWT activity concentrations calculated from the results obtained in each lab are consistent with the overall one confirming that both storage and analytical procedure dedicated to TFWT are fit for purpose.

The ANOVA study in which the lab is the studied factor seems to indicate that the TFWT activity concentrations obtained by lab number 3 and 4 are possibly not consistent with those from the other labs on some measurement day (Fig. 2).

At sample arrival and after one year, results from lab 3 are statistically different $(p<0.05)$. After 1 month $(30 \mathrm{~d})$ of storage, results of lab 4 are statistically different $(p<0.05)$.
However, several experimental points indicate that the bias set in evidence by the ANOVA analysis originates from the fact that participating labs are using different analytical procedures to perform sample dehydration:

- lab 3 and lab 4 dehydration procedures are characterized by higher dispersion with values of about $20 \%$ to be compared to a max value of about $10 \%$ for the other labs;

- TFWT is the tritium fraction which is the most sensitive to isotopic exchange with atmospheric moisture;

- on day 1 corresponding to one of the two high values observed in lab $3\left(34.8 \mathrm{~Bq} \cdot \mathrm{L}^{-1}\right)$, lab 5 one is the lowest (24.5 Bq. $\mathrm{L}^{-1}$ ) observed along the entire exercise in this lab;

- on day 365 corresponding to one of the two high values observed in lab 3 (37.1 Bq. $\mathrm{L}^{-1}$ ), lab 7 one is the lowest (24.5 Bq. $\mathrm{L}^{-1}$ ) observed along the entire exercise in this lab. Moreover, lab 5 value is also below $27 \mathrm{~Bq} . \mathrm{L}^{-1}$;

- on day 30 , lab 5 and 7 values are low $(<27)$, results from all the other labs are very close to 29 (ranking from 28 to 30 ) whereas the one of lab 4 is about 36 ;

- moreover, when the uncertainties associated to individual activity concentration values are considered all the results are consistent.

Following these considerations, it comes that the bias postulate from the ANOVA analysis is more likely due to experimental variations. Therefore, the conclusion issued from mean comparison, that storage conditions are suited, in all participating labs, to preserve sample integrity on a one-year period before TFWT determination are confirmed.

\subsection{Organically bound tritium (OBT)}

Regarding OBT, only seven of the labs whose gave results for TFWT were able to realize the analysis over the one-year period: one lab (lab 4) was under reinstallation after relocation. In addition, the results obtained by lab 3 were clearly overestimated (outlier), so they were not considered here. Two sets of results were obtained: one in Bq.L $\mathrm{L}^{-1}$ of combustion water for labs which are using tubular oven and one in Bq. $\mathrm{kg}^{-1}$ of dry material for all labs. The conversion from Bq.L ${ }^{-1}$ of combustion water to $\mathrm{Bq} . \mathrm{kg}^{-1}$ of dry material 
One factor ANOVA :

Factor $=$ lab
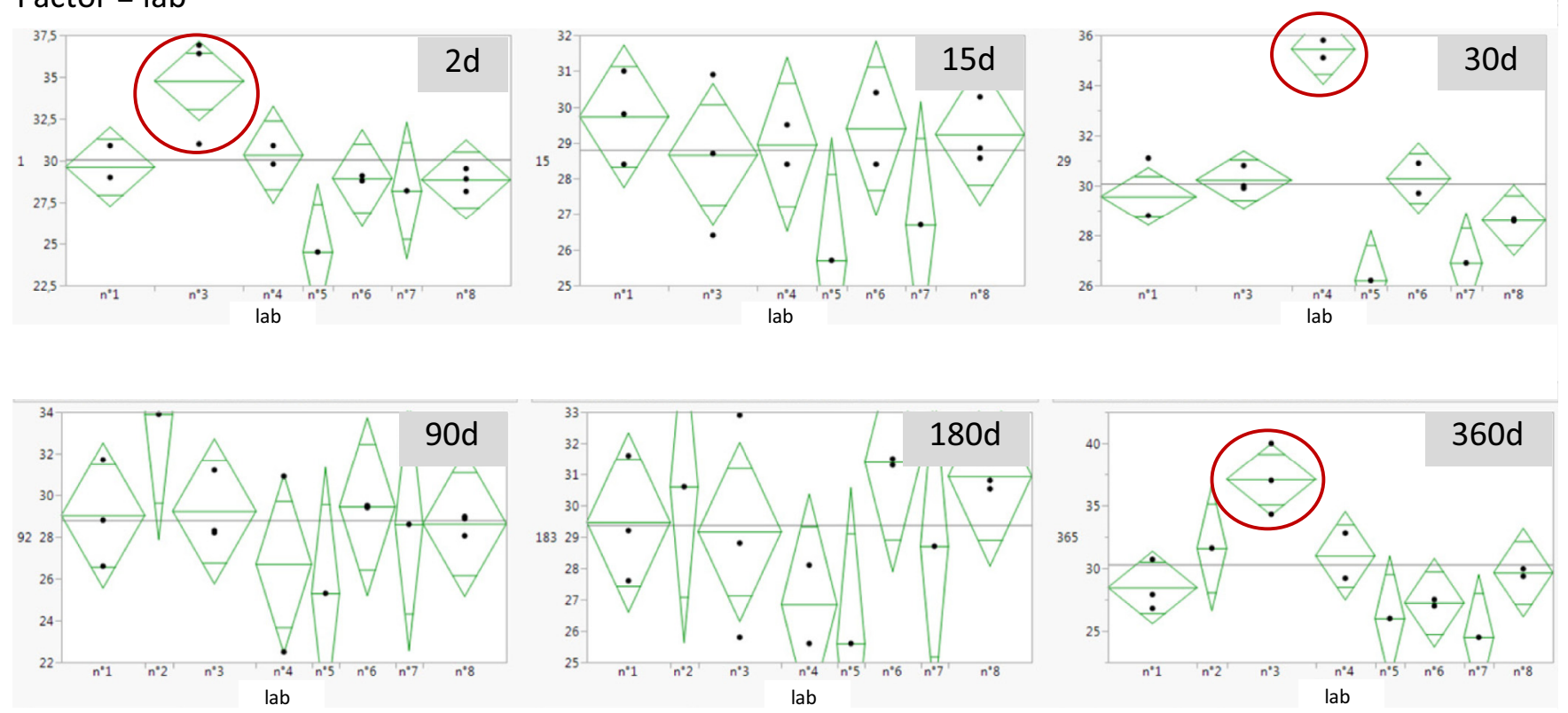

Fig. 2. ANOVA analysis of participating labs activity concentration at each measurement day.

Table 2. OBT activity concentrations (Bq. $\mathrm{kg}^{-1}$ of dry material) determined in each lab for each analysis time plus associated mean ( $\mathrm{m}$ ) and two times the standard deviation (2s) per lab and per day.

\begin{tabular}{|c|c|c|c|c|c|c|c|c|c|}
\hline & $t_{0}+2 \mathrm{~d}$ & $t_{0}+15 \mathrm{~d}$ & $t_{0}+30 \mathrm{~d}$ & $t_{0}+90 \mathrm{~d}$ & $t_{0}+180 \mathrm{~d}$ & $t_{0}+360 \mathrm{~d}$ & m (lab) & $2 \mathrm{~s}$ & $2 \mathrm{~s} / \mathrm{m}(\%)$ \\
\hline Lab 2 \# & 50.0 & 27.0 & 24.0 & 28.0 & & 36.0 & 33.0 & 21.0 & 63.6 \\
\hline Lab 4 & & & & & & 29.3 & 29.3 & & \\
\hline Lab 5 \# & 48.0 & $<36$ & $<42$ & 46.0 & $<34$ & $<33$ & 47.0 & 2.8 & 6.0 \\
\hline Lab 8 & 28.9 & 29.2 & 28.6 & 28.6 & 30.9 & 29.7 & 29.3 & 1.8 & 6.0 \\
\hline m (day) & 36.5 & 28.7 & 29.6 & 32.0 & 29.2 & 30.9 & & & \\
\hline $2 s$ & 19.7 & 2.7 & 7.9 & 14.0 & 3.9 & 5.5 & & & \\
\hline $2 \mathrm{ss} / \mathrm{m}(\%)$ & 54 & 9 & 27 & 44 & 13 & 18 & & & \\
\hline
\end{tabular}

\# labs which are using Oxydiser for which a detection limit (DL) of about 30 to $50 \mathrm{~Bq} \cdot \mathrm{kg}^{-1}$ of dry material is very likely.

depends on multiplicative factor namely the ratio of the hydrogen percentage in the sample to the one in water. The hydrogen percentage of water is known and the one in the sample is determined experimentally. The second one being consistent between the participants only the results in $\mathrm{Bq} \cdot \mathrm{kg}^{-1}$ of dry material will be discussed here after.

Uncertainties obtained by labs for each individual measurement are not quoted in Table 2 where only individual values, mean and standard deviation are presented. In this exercise, for a confidence level of about $95 \%(k=2)$, the relative uncertainties provided for each individual value by participating labs vary from 5 to $70 \%$.

All the data obtained during this one year exercise are summarized in Table 2 for each lab and each analysis time.
Using all the data summarized in Table 2 allows determining the overall mean activity concentration value plus or minus twice its standard deviation: $\overline{\mathrm{C}_{\mathrm{A}}}=(31 \pm 12) \mathrm{Bq} \cdot \mathrm{kg}^{-1}$ (of dry material).

For all the labs, the mean OBT activity concentration calculated from the results obtained at each measurement time are consistent with the overall one. Therefore, storage conditions in all participating lab seem to be appropriate for sample conservation when OBT analysis is concerned. For the two labs which are using an Oxydiser to perform the combustion (\# in Tab. 2), the associated dispersions are higher because the activity concentration present in the sample is about the detection limit achievable by using such an analytical tool. 


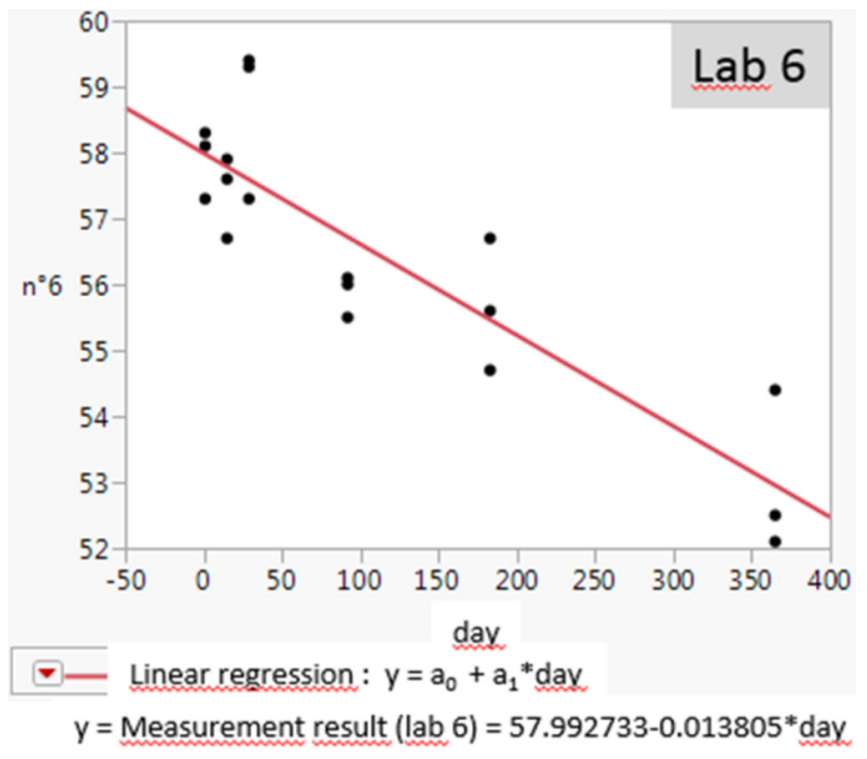

Fig. 3. OBT activity concentration (Bq. $\mathrm{L}^{-1}$ of combustion water) evolution in lab 6 over the one year of the exercise.

Moreover, the ANOVA study seems to indicate that OBT activity concentration obtained by lab number 6 exhibits a decreasing trend all along the one year of the exercise (Fig. 3).

This trend observed by considering lab 6 individual data is less obvious when uncertainties are also taken into account as the max and min values of the confidence intervals are overlapping. Moreover, this trend is observed in only one lab (lab 6) and for OBT determination which is less prone to evolution than TFWT. In addition, relative expended uncertainties $(k=2)$ on OBT activity concentration are generally of about $10 \%$ for a single determination. Another potential source for this decreasing trend is the fact that tritium decay is most of the time not directly considered (about $5 \%$ on a one-year period). Furthermore, in the framework of an international exercise dealing with OBT analysis on wheat it was demonstrated that the sample is stable over a one-year period (Baglan et al., to be submitted).

To confirm the decreasing trend observed in Figure 3 a statistical test was performed. The $\mathrm{F}$ test used here supports this conclusion as the calculated value $\left(F_{\text {calc }}=57.4\right)$ is far higher than the critical one $\left(F_{c}[0.05,1,16]=4.49\right)$. However, there are also several potential experimental origins to explain such an observation.

Anyway, this point should be scrutinized in all future studies aiming to Certified Reference Materials (CRM's) or Reference Materials (RM's) production to ensure sample stability.

The ANOVA study indicates that OBT activity concentration could differ from one lab to another on day 1 and 92 (Fig. 4).

As for a risk $\alpha$ of $5 \%$, the calculated value $\left(F_{\text {calc }}=1.19\right)$ is smaller than the critical one $\left(F_{c}[0.05,5,36] \approx 2.5\right)$, the $H_{0}$ hypothesis that the determined activity concentrations are the same for all measurement days, couldn't be rejected. Anyway, focusing only on statistics it's obvious, that values given by lab 2 on day 1 and 5 on days 1 and 92 are higher than those from the other labs. However, these values correspond to those

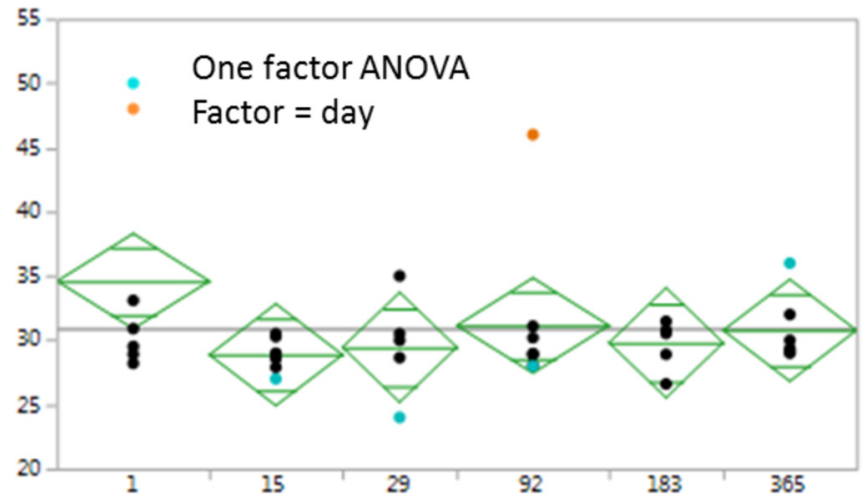

Fig. 4. OBT activity concentration for each measurement day over the one year of the exercise (Lab 2 data are represented by the blue dots and lab 5 ones by the orange dots).

obtained by using an analytical tool at the lower limit of its application range. Indeed, with about one gram of sample combusted by Oxydiser is designed to provide results in short delay after sampling with DL of about $50 \mathrm{~Bq} \cdot \mathrm{kg}^{-1}$ of dry material. Therefore, associated relative expanded uncertainties $(k=2)$ are of about $50 \%$ which demonstrates that when considering all the information these values are consistent with the exercise results. From these results, it looks rather attractive to realize the same exercise with higher activity concentration levels which are sufficient for Oxydiser. However, two main considerations lead to ask about the relevance of such experiments, sample stability is not analyser dependent and Oxydiser are dedicated to rapid analysis for which storage times are reduced.

Therefore, as for TFWT, whatever the time after sampling is, the mean OBT activity concentrations calculated from the results obtained in each lab are consistent with the overall one confirming that both the storage and the analytical procedure dedicated to OBT used by the exercise's participants are suited down to below the $50 \mathrm{~Bq} \cdot \mathrm{kg}^{-1}$ of fresh environmental sample.

\subsection{Uncertainties}

Uncertainty is important regarding the signification of analytical results. In this exercise, participating lab uncertainty budgets were discussed for TFWT and OBT considering on a first approach only the consistency between the different reporting units.

When analysing TFWT, results could be expressed in Bq. $\mathrm{L}^{-1}$ (or Bq.kg ${ }^{-1}$ ) of dehydration water or in Bq.kg ${ }^{-1}$ of fresh material. When using liquid scintillation counting for TFWT activity concentration determination the value obtained for the dehydration water could be converted in Bq. $\mathrm{kg}^{-1}$ of fresh material using the following relation:

$$
A_{\mathrm{TFWT}}^{f}=A_{\mathrm{TFWT}}^{\mathrm{wod}} \times \frac{m_{f}}{m_{\mathrm{wod}}},
$$

with:

$-A_{\mathrm{TFWT}}{ }^{f}$ the tissue free water tritium activity concentration in the fresh material $\left(\mathrm{Bq} \cdot \mathrm{kg}^{-1}\right)$; 
Table 3. Relative expanded uncertainties $(k=2)$ on TFWT activity concentration at each measurement time for both dehydration water $\frac{U_{A_{\mathrm{TFWT}}^{\mathrm{WWd}}}}{A_{\mathrm{TFWT}}^{\mathrm{WoW}}}$ (dw in the table) and fresh material $\frac{U_{A_{\mathrm{TFWT}}^{f}}^{f}}{A_{\mathrm{TFWT}}^{f}}(\mathrm{fm}$ in the table) in \%.

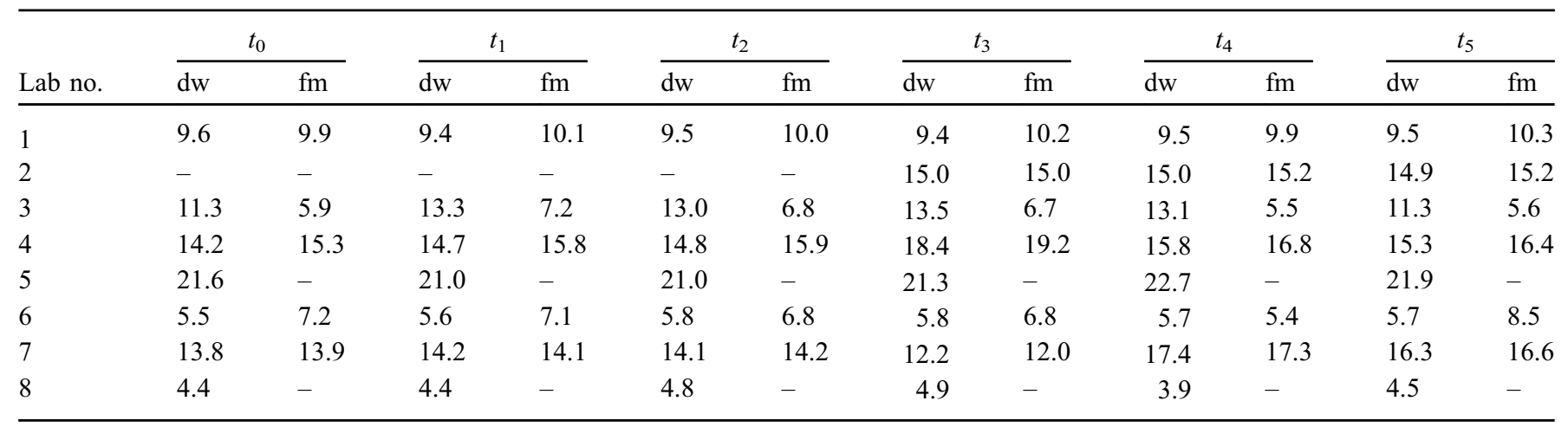

$-A_{\mathrm{TFWT}}{ }^{\text {wod }}$ the tissue free water tritium activity concentration in the dehydration water (Bq. $\mathrm{L}^{-1}$ or Bq. $\mathrm{kg}^{-1}$ );

$-m_{f}$ the mass of fresh material introduced for the dehydration stage $(\mathrm{kg})$;

$-m_{\text {wod }}$ the total mass of dehydration water $(\mathrm{kg})$.

When analysing OBT, results could be expressed in Bq. $\mathrm{L}^{-1}$ (or Bq. $\mathrm{kg}^{-1}$ ) of combustion water, in Bq. $\mathrm{kg}^{-1}$ of dehydrated material or in Bq. $\mathrm{kg}^{-1}$ of fresh material. When using liquid scintillation counting for OBT activity concentration determination the value obtained for the combustion water could be converted in Bq. $\mathrm{kg}^{-1}$ of dehydrated material using the following relation:

$$
A_{\mathrm{OBT}}^{d}=A_{\mathrm{OBT}}^{\mathrm{woc}} \times \frac{\% H_{s}}{\% H_{w}},
$$

with:

- $A_{\mathrm{OBT}}{ }^{d}$ the $\mathrm{OBT}$ activity concentration in the dehydrated material $\left(\mathrm{Bq}_{\mathrm{kg}} \mathrm{kg}^{-1}\right.$ );

- $A_{\mathrm{OBT}}{ }^{\text {woc }}$ the organically bound tritium activity concentration in the combustion water $\left(\mathrm{Bq} . \mathrm{L}^{-1}\right.$ or Bq. $\left.\mathrm{kg}^{-1}\right)$;

- $\% H_{s}$ the hydrogen percentage within the sample (mass);

$-\% H_{w}$ the hydrogen percentage in water (mass).

Once the results is convert to $\mathrm{Bq} \cdot \mathrm{kg}^{-1}$ of dehydrated material, the conversion in Bq. $\mathrm{kg}^{-1}$ of fresh material is performed by using the following relation

$$
A_{\mathrm{OBT}}^{f}=A_{\mathrm{OBT}}^{d} \times \frac{m_{f}}{m_{d}},
$$

with:

- $A_{\mathrm{OBT}}{ }^{f}$ the OBT activity concentration in the fresh material $\left(\mathrm{Bq} . \mathrm{kg}^{-1}\right)$;

$-m_{f}$ the mass of fresh material introduced for the dehydration stage $(\mathrm{kg})$;

$-m_{d}$ the mass of dehydrated material recovered after the dehydration stage $(\mathrm{kg})$.

Without evaluating completely each lab uncertainty budget, some indications could be obtained just by considering the previous relations. For TFWT results, relative uncertainty should be higher when expressed in Bq. $\mathrm{kg}^{-1}$ of fresh material. For OBT, increasing relative uncertainties should be observed from combustion water to fresh material. The experimental results provided by the labs are reported in Table 3 (TFWT), Table 4 (OBT dehydrated material vs. combustion water) and Table 5 (OBT fresh material vs. dehydrated material).

All the data summarized in these tables demonstrate that even if large progresses have been realized for TFWT and OBT determination in environmental samples, improvements are still needed to improve uncertainty budgets. Focusing first on TFWT (Tab. 3), there is no obvious correlation between provided uncertainties and dehydration procedure performance. Moreover, for some labs the uncertainty provided on the fresh material is lower than the one on dehydration water. This last point shouldn't be possible as there are additional components in the one on fresh material regarding dehydration water.

When considering OBT results, similar observation could be done with in addition an inconsistency as uncertainties levels are not increasing constantly from combustion water to dehydrated material and to fresh material (Tabs. 4 and 5). It's difficult to get more information from these data as the main uncertainty component arises from activity concentration measurement and its own influence factors (counting statistic, quench curve or standard addition...) and possibly for OBT from the experimental one on hydrogen percentage. Therefore, works, actions will be promoted to improve analytical labs uncertainty budgets: firstly, to avoid the small inconsistency observed here and secondly to determine which the main components are for both TFWT and OBT activity concentrations (possibly counting statistics, calibration and hydrogen percentage).

\section{Conclusion}

The results obtained during this intercomparison exercise dedicated to storage are very positive while demonstrating some key features:

- storage conditions seem under control for all participating labs;

- with appropriate storage conditions, for both TFWT and OBT, tritium activity concentrations remain stable over a one-year period; 
Table 4. Relative expanded uncertainties $(k=2)$ on OBT activity concentration at each measurement time for both combustion water $\frac{U_{A}^{\mathrm{WBT}}}{A_{\mathrm{OBT}}^{\mathrm{woc}}}(\mathrm{cw}$ in the table) and dehydrated material $\frac{U_{A_{\mathrm{OBT}}^{d}}}{A_{\mathrm{OBT}}^{d}}(\mathrm{dm}$ in the table).

\begin{tabular}{|c|c|c|c|c|c|c|c|c|c|c|c|c|}
\hline Lab no. & \multicolumn{2}{|c|}{$t_{0}$} & \multicolumn{2}{|c|}{$t_{1}$} & \multicolumn{2}{|c|}{$t_{2}$} & \multicolumn{2}{|c|}{$t_{3}$} & \multicolumn{2}{|c|}{$t_{4}$} & \multicolumn{2}{|c|}{$t_{5}$} \\
\hline 1 & 10.0 & 10.9 & 10.1 & 11.0 & 9.9 & 11.0 & 10.1 & 10.9 & 9.9 & 11.1 & 9.9 & 10.9 \\
\hline 2 & - & 28 & - & 48 & - & 75 & - & 50 & - & - & - & 44 \\
\hline 3 & 7.0 & 7.0 & 7.0 & 7.0 & 7.0 & 8.9 & 7.0 & 7.2 & 7.0 & 13.6 & 7.2 & 7.0 \\
\hline 4 & - & - & - & - & - & - & - & - & - & - & 5.6 & 6.1 \\
\hline 7 & 10.1 & 6.0 & 10.1 & 6.1 & 10.1 & 6.0 & 10.1 & 6.1 & 10.1 & 7.9 & 13.0 & 6.9 \\
\hline 8 & - & 4.9 & - & 4.2 & - & 3.4 & - & 4.1 & - & 4.3 & - & 5.4 \\
\hline
\end{tabular}

Table 5. Relative expanded uncertainties $(k=2)$ on OBT activity concentration at each measurement time for both dehydrated material $\frac{U_{A_{\mathrm{O}}^{d}}^{d}}{A_{\mathrm{OBT}}^{d}}$ (dm in the table) and fresh material $\frac{U_{A_{\mathrm{OBT}}^{f}}}{A_{\mathrm{OBT}}^{f}}(\mathrm{fm}$ in the table).

\begin{tabular}{|c|c|c|c|c|c|c|c|c|c|c|c|c|}
\hline \multirow[b]{2}{*}{ Lab no. } & \multicolumn{2}{|c|}{$t_{0}$} & \multicolumn{2}{|c|}{$t_{1}$} & \multicolumn{2}{|c|}{$t_{2}$} & \multicolumn{2}{|c|}{$t_{3}$} & \multicolumn{2}{|c|}{$t_{4}$} & \multicolumn{2}{|c|}{$t_{5}$} \\
\hline & $\mathrm{dm}$ & $\mathrm{fm}$ & $\mathrm{dm}$ & $\mathrm{fm}$ & $\mathrm{dm}$ & $\mathrm{fm}$ & $\mathrm{dm}$ & $\mathrm{fm}$ & $\mathrm{dm}$ & $\mathrm{fm}$ & $\mathrm{dm}$ & $\mathrm{fm}$ \\
\hline 1 & 10.9 & 9.5 & 11.0 & 10.4 & 11.0 & 10.4 & 10.9 & 9.9 & 11.1 & 9.9 & 10.9 & 9.8 \\
\hline 3 & 7.0 & 6.8 & 7.0 & 6.7 & 8.9 & 9.1 & 7.2 & 7.2 & 13.6 & 7.4 & 7.0 & 7.0 \\
\hline 4 & - & - & - & - & - & - & - & - & - & - & 6.1 & 6.7 \\
\hline 5 & 56 & 53 & - & - & - & - & 63 & 60 & - & - & - & - \\
\hline 7 & 6.0 & 8.1 & 6.1 & 8.1 & 6.0 & 8.1 & 6.1 & 8.0 & 7.9 & 8.9 & 6.9 & 8.4 \\
\hline 8 & - & 4.9 & - & 4.2 & - & 3.4 & - & 4.1 & - & 4.3 & - & 5.4 \\
\hline
\end{tabular}

- a good consistency in activity concentration results independently from the analytical tool, even for the one used close to the limits of its application range.

Moreover, the activity concentration level in the studied grass sample is low enough to fit with environmental standards and to demonstrate that when labs have good skills all along the analytical path starting with sample uptake, following with sample storage, sample treatment and finishing with measurement, the obtained results should be consistent. In addition, the dispersion of the results is lower than those observed in previous exercises (CETAMA, 2008, 2010) with higher activity concentrations, demonstrating the improvement made for TFWT and OBT analysis since 10 years.

However, several points need to be improved and validated in the future. Indeed, it should be demonstrated unambiguously if the decreasing trend observed over one year in one lab is due to sample evolution or simply reflect tritium decay. In the same way, works should be done, focusing on uncertainty budget to determine both for TFWT and OBT the main contributors in the combined uncertainty.
Ultimately, all these results and investigations are also part of a larger study, namely to prepare and to validate the use of an environmental matrix as CRM or RM for tritium fractions determination.

\section{References}

AGIR. 2007. Review of risks from tritium. Advisory group on ionising radiation, documents of the Health Protection Agency: radiation, chemical and environmental hazards. REC 4. November 2007. Available from http://www.hpa.org.uk/ publications.

ASN. 2010. Livre blanc du tritium. Paris : Autorité de Sureté Nucléaire.

Baglan N, Ansoborlo E, Cossonnet C, Fouhal L, Deniau I, Mokili M, Henry A, Fourré E, Olivier A. 2010. Métrologie du tritium dans différentes matrices: cas du tritium organiquement lié, (TOL), Radioprotection 45(3): 369-390.

Baglan N, Kim SB, Cossonnet C, Croudace IW, Fournier M, Galeriu D, Warwick PE., Momoshima N, Ansoborlo E. 2013. Organically bound tritium (OBT) behaviour and analysis: outcomes of the seminar held in Balaruc les Bains in May 2012, Radioprotection 48: 127. 
Baglan N, Cossonnet C, Roche E, Kim SB, to be submitted

Belot Y, Roy M, Métivier H. 1996. Le tritium de l'environnement à l'homme. Les Ulis (France) : Les Éditions de Physique.

Brudenell AJP, Collins CD, Shaw G. 1997. Dynamics of tritiated water (HTO) uptake by crops after short-term atmospheric release, J. Environ. Radioact. 36: 197.

CETAMA. 2008. Circuit intercomparaison 2005-2008, Analyse du tritium organiquement lié dans une matrice herbe, Commission d'établissement des méthodes d'analyse-DEN/DCRP/ CETAMA/NT/2008/05.

CETAMA. 2010. Circuit intercomparaison 2009-2012, Analyse du tritium organiquement lié dans une matrice herbe, Commission d'établissement des méthodes d'analyse-DEN/DCRP/ CETAMA/NT/2010/03.
CETAMA. 2013. Méthode 384 : Analyse du tritium dans les matrices environnementales. Commission d'établissement des méthodes d'analyse DEN/DRCP/CETAMA/NT/2013/03.

Keum DK, Lee HS, Kang HS, Jun I, Choi YH, Lee CW. 2006. Prediction of tritium level in agricultural plants after short-term exposure to HTO vapor and its comparison with experimental results, Health Phys. 90(N1): 42.

Kim SB., Baglan N, Davis PA. 2013. Current understanding of organically bound tritium (OBT) in the environment, J. Environ. Radioact. 126: 83.

König LA. 1990. Tritium in the food chain, Radiat. Prot. Dosim. 30(2): 77-86. Pointurier F, Baglan N, Alanic G. 2004. A method for the determination of low level organic-bound tritium activities in environmental samples, Appl. Radiat. Isot. 61: 293-298.

Cite this article as: Baglan N, Ansoborlo E, Cossonnet C, Losset Y, Crozet M. 2017. Investigation of the potential impact of storage place on tissue free water tritium and organically bound tritium activity determination. Radioprotection 52(4): 281-289 